\title{
The regulation and functions of the nuclear RNA exosome complex
}

Cornelia Kilchert ${ }^{1}$, Sina Wittmann ${ }^{1}$ and Lidia Vasiljeva ${ }^{1 \S}$

${ }^{1}$ Department of Biochemistry, University of Oxford, South Parks Road, OX1 3QU, UK

§To whom correspondence should be addressed:

Department of Biochemistry, University of Oxford, South Parks Road, OX1 3QU, UK. Tel.: +44 1865613352; Fax:+44 1865275399; E-mail: lidia.vasilieva@bioch.ox.ac.uk

\begin{abstract}
The RNA exosome complex is the most versatile RNA degradation machine in eukaryotes. The exosome has a central role in multiple aspects of RNA biogenesis, including in RNA maturation and surveillance. Moreover, it is emerging as an important player in regulating expression levels of specific mRNAs in response to environmental cues and during cell differentiation and development. While the mechanisms of RNA targeting to (or escape from) the exosome are still not fully understood, general principles begin to emerge, which we discuss in this review. In addition, we introduce and discuss novel, previously unappreciated functions of the nuclear exosome, including in transcription regulation and in the maintenance of genome stability.
\end{abstract}

\section{Introduction}

The RNA exosome complex was initially identified as a central factor in the processing of stable RNA species produced by RNA Polymerase I, II, and III (Pol I, II and III) such as rRNAs, small nuclear (sn) RNAs, small nucleolar (sno) RNAs and tRNAs ${ }^{1-4}$. Since then, 
targets of the exosome complex have been catalogued genome-wide in yeast, flies, mice, humans and plants ${ }^{5-11}$, revealing a wide variety of substrates. In all eukaryotic cells, the exosome plays a central role in RNA quality control.

An important function of the exosome is the removal of RNAs that arise as a result of cryptic transcription ${ }^{10,12-19}$. "Cryptic" is the name given to the totality of RNA species that are highly unstable in wild-type cells and therefore can only be detected when nuclear RNA surveillance is compromised ${ }^{14,15,20,21}$. In large part, these cryptic unstable transcripts (or CUTs, as they are known in yeast) are derived from transcription at bidirectional promoters, where antisense transcripts termed promoter upstream transcripts (PROMPTs) in humans or upstream antisense RNA (uaRNA) in mouse, are rapidly degraded by the nuclear exosome ${ }^{12,13,20,22}$. Similarly, mammalian enhancers give rise to unstable bidirectional transcripts, which are commonly referred to as enhancer RNAs (eRNAs) and are also targeted by the exosome ${ }^{10,18,19}$. Heterochromatin-forming repetitive elements, such as rDNA repeats and centromeres, are another source of unstable transcripts ${ }^{21,23-25}$. Although cryptic transcripts are commonly short, many intergenic or antisense long noncoding RNAs (lncRNAs) are also rapidly degraded by the exosome. Their degradation frequently depends on the same factors that are involved in the destabilization of short cryptic transcripts ${ }^{17}$, which will be described below.

In addition to cryptic transcripts, the exosome removes various aberrant transcripts, including stable RNA species that are incorrectly processed and mRNAs that fail to undergo proper splicing or 3'end formation ${ }^{7,8,10,26-28}$. Accumulation of aberrant RNA species is harmful because they can compete with properly processed RNAs for factors. For example, accumulation of unprocessed snRNAs has been associated with defective splicing ${ }^{29}$. Moreover, having “too much” RNA poses a threat in itself: Accumulation of exosome substrates in the cytoplasm activates anti-viral defence mechanisms and can trigger 
autoimmunity ${ }^{30}$. In the nucleus, excess RNA can hybridize with homologous genomic loci, forming RNA-DNA hybrids ${ }^{31}$. These can induce DNA double strand breaks (DSBs) and are associated with increased genomic instability.

In addition, recent studies have revealed that the exosome regulates the levels of specific transcripts in response to environmental cues, and is required to orchestrate genetic programmes during development ${ }^{32-36}$. Given all these functions it is not surprising that the exosome complex is essential. Deregulation of the exosome leads to severe neurological diseases such as spinal muscular atrophy and pontocerebellar hypoplasias ${ }^{37,38}$. In this review, we summarize the roles of the exosome complex in the nucleus. First, we give a short description of the structure of the complex, which has been covered in detail in excellent recent reviews ${ }^{1,39}$. We then introduce important co-factors and delineate known mechanisms of exosome recruitment to RNA. We outline the nuclear pathways of mRNA quality control and describe how the exosome is harnessed to regulate mRNA levels. Finally, we discuss recent work describing potential new roles for the RNA exosome, for example in transcription regulation, in the removal of RNA-DNA hybrids and in the DNA damage response (DDR).

\section{The exosome complex and its regulation}

The exosome acts on all classes of cellular RNAs at various stages of their existence, from synthesis to maturation and turnover. One of the long-standing questions in the field is how the exosome complex can be targeted to such a diversity of substrates. This is now known to involve a variety of co-factors that recognize the RNA substrate and help to initiate its degradation by the exosome complex. 
The exosome complex. The eukaryotic RNA exosome is an evolutionarily conserved ribonucleolytic complex that consists of ten to eleven subunits (Figure 1A). The barrelshaped nine-subunit core (known as EXO9) is catalytically inactive and comprises the 'cap' structure, which is formed of the three proteins ribosomal RNA processing protein 4 (Rrp4), Rrp40 and Csl4, which contain both the S1 and the KH RNA-binding domains, and the 'ring' complex, which is comprised of RNase PH-like proteins Rrp41, Rrp42, Rrp43, Rrp45, Rrp46 and Mtr3. The core associates with the processive 3' to 5' exo- and endoribonuclease Dis3 (also known as Rrp44) at the bottom of the PH-protein barrel and/or with the distributive 3' to 5' exonuclease Rrp6 (also known as exosome component 10 or PM/Scl-100 in humans) at the cap-side, forming the exosome complexes $\mathrm{EXO}^{\mathrm{Dis} 3}, \mathrm{EXO}^{\mathrm{Rrp} 6}$ or EXO11 ${ }^{\text {Dis3+Rrp6 }}$, respectively ${ }^{1,39,40}$. Although some RNAs can be targeted directly to Dis3 or Rrp6, the majority of substrates enter the barrel-like structure of the exosome via a pore at the centre of the S1/KH cap and are threaded through the central channel in a 3' to 5' orientation to access the active centre of Dis $3^{41-44}$. The endonuclease activity of Dis3 facilitates the degradation process ${ }^{7,45,46}$. Although Dis3 is active in isolation, all subunits of EXO10 ${ }^{\text {Dis3 }}$ are essential for viability in Saccharomyces cerevisiae ${ }^{3}$, suggesting that the requirement for substrates to traverse the channel is important to control the activity of the catalytic subunit. In addition, Rrp6 can bind the complex EXO10 ${ }^{\text {Dis3 }}$ to form EXO11 $1^{\text {Dis3+Rrp6 }}$. In that case, the RNA can either be threaded through the channel to reach Dis3 or it can be degraded by Rrp6. The path the RNA takes to Rrp6 is currently not clear. Based on a recent crystal structure it was proposed that the RNA reaches the active site of Rrp6 directly, without making contacts to any of the other exosome subunits (Figure $1 \mathrm{~B})^{47}$. However, a different route has also been suggested: Crystallographic evidence together with UV crosslinking experiments indicated that the 3' end of the RNA could enter from the side - between the cap and the ring - and then traverse the cap into the Rrp6 active site ${ }^{44,48}$. Which model is correct, or if indeed, both 
routes are utilized in vivo has yet to be determined. In the case of very structured, bulky RNAs, Rrp6 was shown to detach from the core and swing away from the channel, which allows the substrate to enter the channel directly ${ }^{47}$. It was also demonstrated that Rrp6 can allosterically stimulate the nucleolytic activity of Dis3 in budding yeast ${ }^{48}$, probably by direct RNA binding as well as widening of the channel ${ }^{44,47}$. Which exonuclease is selected in vitro appears to be stochastic and the substrate is repeatedly bound and released until the processive Dis3 is reached, which then degrades the RNA to completion. The path taken might be influenced by the structure of the RNA substrate as well as by cofactors bound to it $^{44}$.

Rrp6, Dis3 or the Dis3-like exonuclease DIS3L in higher eukaryotes interact with EXO9 to form distinct complexes with discrete subcellular localization (Figure 1C) $39,40,49$. These exosome isoforms are proposed to carry out specialized functions in different cellular compartments. In this review, we will mainly focus on the functions of the nuclear exosome.

Regulators of exosome activity. On its own, the exosome will degrade any RNA, provided it is not too structured. However, the activity of the isolated complex is comparatively weak $^{3,50,51}$. Interestingly, in vitro, substrate turnover by Dis3 is significantly increased in the absence of the EXO9 core ${ }^{48}$. In addition, the length of unstructured sequence at the 3' end required for Dis3 to be able to degrade an RNA drops from 30-35 bp to 7-9 bp in the absence of EXO9 ${ }^{51,52}$, demonstrating that the long central channel restricts substrate access to the nuclease. Befittingly, it is becoming increasingly apparent that RNA-unwinding activities are central to the regulation of the exosome, as they facilitate threading of the RNA substrate through the narrow channel entrance. In S. cerevisiae, two related conserved DExH-box helicases, Mtr4 and Ski2, are required for RNA degradation in the nucleus and the cytoplasm, respectively ${ }^{53,54}$. Both helicases are associated with accessory factors. Mtr4, for example, interacts with a non-canonical polyA polymerase (Trf4 or Trf5) and a Zn-knuckle RNA- 
binding protein (Air1 or Air2) to form the Trf4/5-Air1/2-Mtr4 polyadenylation complex (TRAMP) $^{55}$. This complex facilitates degradation of substrates by the exosome: Addition of short unstructured polyA tails by Trf4 or Trf5 is thought to provide "grip" for Mtr4, which then unwinds the RNA and feeds it into the exosome complex ${ }^{17,56}$.

The central role of Mtr4 in nuclear RNA turnover is widely conserved. However, apart from S. cerevisiae, most other organisms seem to have developed a division of labour between a nucleolar complex, which is associated with a polyA polymerase, and a nucleoplasmic complex, which is not (Table 1). In humans, for example, MTR4 (also known as SKIV2L2) associates with two such separate complexes: In the nucleoplasm it is part of the nuclear exosome targeting (NEXT) complex, which mediates decay of PROMPTs and eRNAs and controls levels of snRNAs precursors ${ }^{18,57,58}$. The second complex resembles yeast TRAMP and contains the polyA polymerase TRF4-2, but is exclusively nucleolar and predominantly involved in rRNA processing ${ }^{18}$. In the fission yeast Schizosaccharomyces pombe, Mtr4 associates with the polyA polymerase Cid14 and is also enriched in the nucleolus, where it is involved in the processing of rRNA intermediates and the turnover of heterochromatic RNA $^{21,25,59}$. Fission yeast TRAMP is complemented by an additional, Mtr4-like helicase, Mtl1, for the turnover of cryptic transcripts and aberrant mRNAs, which is presumed to take place in the nucleoplasm ${ }^{60-62}$. Similarly, the predominantly nucleolar Arabidopsis thaliana MTR4 (encoded by At1g59760) is aided by a nucleoplasmic helicase, HEN2, which is plantspecific $^{63}$. In addition, other helicases such as the mammalian DEAD-box protein DDX5 and the DEAH-box helicase RHAU are thought to facilitate RNA decay by the exosome complex ${ }^{53}$

Targeting RNA to the exosome. RNA helicases, like the exosome complex itself, are largely unspecific $^{53,54,64,65}$. Thus, selective targeting of the exosome in vivo relies on the ability of primary exosome-specificity factors (ESFs) to recruit the helicases to specific substrates 
(Figure 2A). To date, only a handful of ESFs have been identified. The earliest known example were proteins that bind AU-rich elements (AREs), which are often present in the 3' untranslated regions (UTRs) of unstable mRNAs ${ }^{66}$. ARE-binding proteins (ARE-BPs) are found in all eukaryotes and regulate RNA metabolism. Importantly, they mediate decay of ARE-containing mRNAs by the exosome ${ }^{66}$. In humans, ARE-BPs recruit the DEAH-box helicase RHAU, which interacts with the exosome complex and enhances ARE-dependent RNA decay (Figure 2B) ${ }^{67}$.

The nucleolar rRNA processing factors Utp18 and Nop53 in S. cerevisiae were recently shown to recruit TRAMP to different rRNA processing intermediates ${ }^{68}$. Both proteins contain a conserved arch interaction motif (AIM), which directly binds to the conserved arch domain of Mtr4. Also in S. cerevisiae, the trimeric complex NNS, which is comprised of the RNAbinding proteins Nrd1 and Nab3 and the helicase Sen1, recognizes sequence elements that are enriched on unstable Pol II-transcribed ncRNAs. NNS also associates with snRNAs and snoRNAs that are known to undergo 3' processing by the exosome complex ${ }^{69-71}$. Through its interaction with Pol II early during the transcription process, NNS mediates transcription termination and hands the transcripts over to Mtr4-containing TRAMP complexes (Table 1),

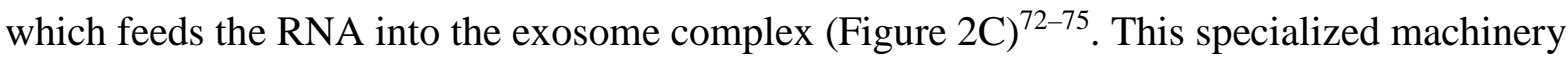
for transcription termination-coupled decay appears to be unique to budding yeast. In contrast, human PROMPTs, which rely on the conventional polyadenylation and cleavage machinery for transcription termination ${ }^{76,77}$, are nevertheless targeted to the exosome by NEXT (Table 1) $)^{18,57}$. The exact mechanism, however, remains unclear.

In S. pombe, the RNA-binding protein Mmi1 is co-transcriptionally recruited to sequence elements known as "determinants of selective removal” (DSRs), which are enriched on meiotic mRNAs ${ }^{32,78}$. Mmi1 binding programs transcripts for rapid exosome-dependent decay $^{32,79,80}$ and - among other proteins - recruits the helicase Mtl1, which is required for 
transcript turnover ${ }^{60,62}$. The Mmi1 pathway is essential to suppress the activation of the meiotic programme during mitotic growth, but has also been found to mediate degradation of regulatory ncRNAs ${ }^{32,34,35,81}$. Intriguingly, Mmi1 binding to RNA involves its conserved YTH domain $^{82}$. Proteins of the YTH family have recently attracted interest because they were shown to selectively bind N6-methylated adenosines on RNA ${ }^{83}$. The residues involved in methyl recognition are conserved in $\mathrm{Mmi1}^{84}$ and Mmi1 binding overlaps with mapped sites of N6-methyl adenosine on snRNAs (C. Kilchert, S. Granneman and L. Vasiljeva, unpublished results), raising the possibility that nuclear RNA decay by the exosome could be regulated by RNA modifications.

Altogether, the ability of ESFs to recognize specific RNA sequence elements, secondary structures and/or RNA modifications allows cells to distinguish between different RNA species. It has to be noted that although the known ESFs recognize sequences that are very common in the genome, actual binding often appears to be more selective $\mathrm{e}^{16,35}$. This suggests cooperativity for RNA-binding between different ESFs or components of ESF complexes. In addition, cooperative binding of multiple elements, exemplified by the dual affinity of Nrd1 for early elongating Pol II and RNA sequence elements (Figure 2C) ${ }^{72,85}$, may allow for selective targeting. Combined, substrate recognition by ESFs and the selective recruitment of RNA helicases that facilitate RNA degradation form the basis for the regulatory functions of the exosome complex.

Negative regulators of exosome function. There are two different ways in which the activity of the exosome can be negatively regulated. First, factors that have a general RNA stabilizing capacity can bind RNA to prevent targeting to the exosome. Such a role was described for the nuclear polyA-binding protein Nab2 in S. cerevisiae, which is recruited early during mRNA biogenesis and prevents decay by the exosome ${ }^{86,87}$. Other substrates are only partially degraded by the exosome complex, for example during 3'end processing of stable rRNAs, 
snRNAs and snoRNAs ${ }^{4,88}$. Here, it is generally believed that the presence of protective sequence features shields stable transcripts from complete decay, although direct experimental evidence is scarce. For example, the $\mathbf{S m}$ ring, which is a stable heptameric protein complex, binds at the 3' ends of mature snRNAs and of yeast telomerase RNA and is thought to physically impede RNA degradation by the exosome. Insertion of a Sm binding site was shown to stabilize the unstable non-coding transcript $\mathrm{NTS}^{29}$. In contrast, human telomerase RNA is protected by a snoRNA-like domain at its 3'end ${ }^{89}$. snoRNA-like structures also stabilize several human "sno-IncRNAs ${ }^{90}$. This and other mechanisms that allow RNAs to escape from degradation by the exosome are the focus of a recent review ${ }^{91}$.

\section{Exosome roles in mRNA quality control}

To yield functional transcripts, mRNAs need to be extensively processed. Like many complex biological processes, RNA processing is intrinsically error-prone and can result in the production of dysfunctional and potentially toxic molecules. The exosome complex has a central role in nuclear mRNA quality control, and aberrant mRNA species accumulate massively upon its depletion. However, the mechanisms that underlie exosome targeting to defective mRNAs are not well understood. In particular, the timing of quality control relative to RNA synthesis is uncertain. In contrast to the cytoplasm, where exosome-dependent degradation takes place in the context of translation, nuclear RNA decay is intimately linked to transcription. There is a general notion that susceptibility to degradation by the nuclear exosome is a fate that is set co-transcriptionally $y^{71,92,93}$. It is currently unclear whether the triggering of decay following the recognition of an error is also limited to this narrow temporal window, or whether nuclear mRNA quality control can function posttranscriptionally. 
Quality control of 3'end processing. One type of transcripts that are co-transcriptionally targeted to the exosome are those in which transcription fails to terminate at the polyadenylation site (PAS). In S. cerevisiae, such read-through transcription frequently terminates through non-conventional mechanisms, for example at transcription roadblocks constituted by DNA-binding proteins such as Reb1, at sites that are recognized by the NNS complex, or following cleavage of the nascent RNA by the RNase III enzyme Rnt1, which acts on double-stranded RNA. Such non-conventional termination events are coupled to rapid RNA decay by the exosome complex ${ }^{94-97}$. Sites non-conventional termination are enriched downstream of genes and are thought to act as "fail-safes" that protect neighbouring genes from transcriptional interference ${ }^{98}$.

Splicing-coupled quality control. An important class of substrates for the nuclear exosome are pre-mRNAs with retained introns ${ }^{7,8,28,99}$. Accumulation of pre-mRNAs in exosome mutants is frequently accompanied by an increase in spliced transcript, suggesting that at least some of the pre-mRNAs targeted by the exosome have the potential to complete splicing ${ }^{8,28}$. Hence, it has been proposed that splicing and the quality control pathways that lead to transcript degradation by the exosome kinetically compete for their pre-mRNA substrates. Slow splicing leads to degradation of a large proportion of pre-mRNAs and as a result, to less mRNA production, whereas faster splicing converts more pre-mRNA into mRNAs. However, how decay is initiated on pre-mRNAs remains obscure. Across organisms, nuclear polyA-binding proteins have been implicated in pre-mRNA turnover, and can in some cases also affect splicing efficiency ${ }^{86,92,99-102}$. In addition, at least in human cells, susceptibility to nuclear polyA-binding protein-mediated decay appears to correlate with inefficient nuclear export and prolonged nuclear retention ${ }^{103}$. In S. cerevisiae, unspliced premRNAs are bound by the SR proteins Gbp2 and Hrb1, which prevent their nuclear export $^{92,104}$. In the absence of these factors, pre-mRNAs with retained introns evade 
degradation by the exosome and escape into the cytoplasm ${ }^{104}$. Splicing or at least commitment to splicing is now thought to occur co-transcriptionally in most organisms, and cross-talk with quality control factors has been observed ${ }^{105,106}$. In budding yeast, for example, co-transcriptional recruitment of the TRAMP complex to introns was found to stimulate splicing ${ }^{106}$. In S. pombe and humans, there is evidence for direct interactions of exosome cofactors with the spliceosome, and disruption of these interactions leads to an increase in premRNA levels ${ }^{60,61,107}$. Together, this suggests that splicing and mRNA quality control are an integrated process. However, a clear picture of its regulation has yet to emerge.

Quality control of RNA packaging. The exosome also degrades mRNAs with defects in cotranscriptional packaging into ribonucleoprotein particles (mRNPs), for example in S. cerevisiae cells with mutations in factors of the THO complex ${ }^{108,109}$. The THO complex is recruited to nascent transcripts and is an important RNA packaging factor that links mRNA transcription with nuclear export. How prevalent packaging defects are in physiological contexts remains unclear, but the genetic links between mRNP biogenesis factors and Rrp6 are strong: Besides transcript destabilization, mutations in THO can result in premature transcription termination and RNA retention at the site of transcription (see below), in a manner dependent on Rrp6 (but not the exosome in general) ${ }^{109,110}$.

Retention of aberrant transcripts on the chromatin. Decay of faulty transcripts is only one aspect of nuclear quality control. Across eukaryotes, release of aberrant transcripts from the chromatin is inhibited ${ }^{109,111-114}$. Retention at the site of transcription appears to be an almost universal response to RNA processing failure, and was observed following defects in diverse maturation processes such as mRNA splicing, 3'end processing, and mRNP formation and export. In all cases, RNA retention on the chromatin was found to depend on Rrp6 ${ }^{109,111-113}$. However, the molecular mechanism remains unclear. Defects were frequently associated with either hyper- or hypo 3' polyadenylation and the retained mRNAs presumably lack 
translation competence. This has been confirmed for mRNAs that accumulate in mutants of the export factor mex67 in S.cerevisiae ${ }^{115}$ : when release of the retained mRNAs was forced by overexpression of export factors, no protein was produced. In contrast, retained mRNAs which were released from the chromatin over time were translationally active, indicating that they had the potential to mature into functional mRNPs ${ }^{115}$. Thus, retention of aberrant mRNA at the chromatin may serve a dual purpose: It prevents release of faulty transcripts into the cytoplasm, while possibly providing transcripts with compromised processing additional time to complete their maturation. Susceptibility to decay by the exosome and retention at the chromatin are both features that are more typically associated with ncRNAs ${ }^{116}$, and it is striking that mRNAs with processing defects appear to acquire a ncRNA fate. For some ncRNAs, Rrp6-dependent tethering to the chromatin has been convincingly linked to their function. One notable example is from recent work in A. thaliana, where RRP6L1 - one of three Rrp6-like proteins in plants — was found to be involved in tethering a Pol Vtranscribed scaffold RNA to the chromatin, where it serves as a guide for RNA-directed DNA methylation and gene suppression ${ }^{117}$.

\section{Regulation of gene expression}

Orchestrated changes in mRNA stability play an important role in enabling transitions between different metabolic states and the quick adaptation to external cues ${ }^{118}$. Previously, it has been assumed that modulation of mRNA stability is primarily coordinated in the cytoplasm. However, it has now become clear that the nuclear exosome also targets and represses the expression of specific genes or complete genetic programmes.

Regulation of decay by $\mathbf{m R N A}$ processing. A striking case of regulated mRNA decay is the Mmi1-dependent degradation of meiotic mRNAs during mitotic growth in S. pombe, which 
prevents the untimely induction of meiosis (Figure 2D). When cells enter meiosis, Mmi1 is sequestered in nuclear foci. This abrogates Mmi1-dependent decay and results in stabilization of meiotic transcripts ${ }^{32}$. Another example comes from S. cerevisiae. Here, Rrp6p is known to rapidly degrade mRNAs that encode replication-dependent histones as cells leave the S phase. The process is TRAMP-dependent, but the mechanism of TRAMP targeting remains unknown (Table 1$)^{119,120}$. Interestingly, decreased RNA stability is frequently a consequence of changes in mRNA processing (Figure 3). S. cerevisiae NRD1 mRNA, for example, contains multiple NNS-binding sites ${ }^{121}$. If levels of Nrd1 are low, transcription of the mRNA terminates normally and it is stably expressed. However, if Nrd1 levels are high, recruitment of the NNS complex to NRD1 mRNA induces premature termination, thus reducing gene expression in an auto-regulatory feedback loop ${ }^{121}$ (Figure 3A). Other mRNAs were also suggested to be repressed by premature termination ${ }^{122}$. mRNAs that contain cryptic introns represent another example. These are generally spliced at low rates, but splicing can be activated in response to environmental cues. Splicing of cryptic introns can be coupled to RNA decay by the exosome (Figure 3A), which is then referred to as spliceosome-mediated decay (SMD) ${ }^{123}$. For example, the bromodomain protein Bdf1 in S. cerevisiae facilitates recruitment of the spliceosome to a cryptic splice site in the BDF2 mRNA. In striking contrast to the conventional scenario, where splicing generates functional mRNA, splicing products here are rapidly degraded by the exosome complex thereby reducing the levels of functional (unspliced) BDF2 transcript ${ }^{123}$. For Bdf2, this mechanism is particularly active during the $\mathrm{DDR}^{124}$. SMD was shown to repress several genes in S. cerevisiae ${ }^{123}$. In addition, recent studies in S. pombe identified cryptic introns in many exosome targets, suggesting that the mechanism might be conserved ${ }^{27,60}$.

Both activation of premature termination and SMD represent cases where the unprocessed transcripts are functional protein-coding mRNAs, whereas RNA processing induces RNA 
decay (Figure 3A). This novel role for RNA processing as a negative regulator of gene expression is opposed to its classical positive contribution to gene expression by generating functional mRNAs. Conversely, transcript decay by the exosome can also be induced by suppression of mRNA processing. This exploits the stringent nature of nuclear quality control, which ensures that unprocessed mRNAs are rapidly degraded (Figure 3B). Negative regulation of mRNA processing can act at several stages during mRNA maturation, but regulated intron retention and inhibition of 3'end processing have been most frequently reported $^{33,96,99,100,125,126}$. It is striking that suppression of mRNA processing is particularly prevalent among the transcripts of RNA-binding proteins, which frequently destabilize their own transcripts in auto-regulatory feedback loops. For example, recruitment of Rpl9 to a structural element in the $R P L 9 b$ mRNA prevents efficient transcription termination in S. cerevisiae ${ }^{96}$. The read-through product is terminated at a downstream NNS-dependent site and is rapidly degraded by the exosome. The polyA-binding protein Nab2 is also involved in the 3'end processing of its own mRNA ${ }^{127}$.

Coordination of intron retention and pre-mRNA turnover. Auto-regulation of other RNA surveillance factors, such as the human nuclear polyA-binding protein PABN1 or the RNA helicase Dbp2 and the mRNA export factor Yra1 in S. cerevisiae, involves intron retention ${ }^{100,125,126}$. In yeast, multiple genes are duplicated, and expression of paralogous genes is frequently controlled by negative feedback regulation. For example, splicing of mRNAs that encode ribosomal proteins was shown to be repressed in the presence of the paralogous protein, which presumably binds the pre-mRNA to interfere with RNA processing ${ }^{99,128-130}$. In several cases, paralogue-mediated inhibition of splicing was shown to be coupled to RNA decay by the exosome $e^{35,99}$ (Figure 3B). During cellular differentiation, co-ordinated intron retention in multiple transcripts has been observed in human cells, resulting in pre-mRNA 
decay in the cytoplasm or the nucleus ${ }^{33,131-133}$. Here, negative regulation of RNA processing is central to the efficient execution of genetic programmes.

The fraction of pre-mRNAs that is degraded by the nuclear exosome under normal growth conditions is substantial ${ }^{7,8}$. In S. cerevisiae dis3 mutants, $49 \%$ of intronic sequences were found to be upregulated. In $46 \%$ of cases where the pre-mRNA was stabilized, levels of the spliced transcript also increased ${ }^{8}$. Why intron-containing mRNAs are subject to such high levels of turnover has been a matter of debate. Deletion of introns in yeast genes frequently resulted in reduced fitness under stress ${ }^{134}$, indicating that their presence confers an advantage during cellular adaptation. We suggest that splicing efficiency at many genes could be finetuned to render introns "sensitive to quality control” (Figure 3C). Futile cycles of RNA synthesis and decay could provide the cell with great regulatory flexibility, as slowing down splicing by merely a fraction could, through subjection of the RNA to decay, considerably reduce expression levels of an intron-containing gene.

Interestingly, introns sometimes contain features that render them particularly sensitive to quality control. The introns of $R P L 18 A$ and $R P L 22 B$ in S. cerevisiae, for example, contain an RNase III cleavage site which renders the pre-mRNAs unstable and restricts expression of both genes ${ }^{135}$. In S. pombe, Mmi1 is recruited to several intron-containing genes, including rps2202, the homologue of $R P L 22 B$. Mmi1 binding induces rapid exosome-mediated decay of the pre-mRNA and limits the level of spliced transcript. Under conditions where splicing occurs rapidly, Mmi1 is no longer recruited and gene repression is relieved ${ }^{35}$. We refer to such quality control-hypersensitive introns as “decay-promoting” and suggest that they are associated with genes that require particularly tight regulation.

\section{Additional functions of the exosome}


Mutation in exosome components can result in very complex phenotypes. Recent studies have identified several functions for the exosome complex that go beyond its well-known role in the post-transcriptional regulation of RNA stability, for example it has been implicated in the regulation of transcription and in the maintenance of genome integrity.

Transcription. Recent studies suggest that the exosome complex may play a role in transcription termination. In S. cerevisiae, Rrp6 is required for the recruitment of Nrd1 to the PHO84 locus and for early, NNS-dependent transcription termination of a regulatory antisense transcript produced from the locus ${ }^{136}$. A recent genome-wide study identified multiple transcripts in budding yeast that depend on Rrp6 for transcription termination ${ }^{137}$. In S. pombe, Rrp6 is required for proper termination of a regulatory ncRNA, but not of the overlapping pho1 mRNA that shares the same PAS with it ${ }^{34}$. Although this observation is intriguing, the mechanism of transcription termination of the ncRNA remains unclear. Also in fission yeast, depletion of Dis3 led to the accumulation of mRNAs with extended 3' ends and increased Pol II association with chromatin downstream of PASs ${ }^{138}$. Here, it was proposed that Pol II might stall, backtrack, and thus expose the 3'OH end of the nascent transcript, which would be susceptible to decay by the exosome. This could enable the exosome to displace Pol II from the nascent transcript, causing both transcription termination and transcript degradation. This "reverse torpedo" model is somewhat analogous to the "torpedo" model of transcription termination after PAS cleavage, which posits that degradation of the nascent RNA by a 5' to 3' exonuclease (Rat1 in S. cerevisiae, 5'-3' exoribonuclease 2 (XRN2) in humans) helps to disassociate Pol II from the DNA ${ }^{139,140}$. Exosome depletion had no effect on Pol II levels upstream of the PAS ${ }^{138}$, suggesting that exosome-dependent Pol II displacement acts as a fail-safe rather than a constitutive mechanism for transcription termination. In mammalian cells at least, termination regions of many genes are prone to 
form RNA-DNA-hybrids (“R-loops”, Box 1), which induce Pol II pausing and could favour its backtracking ${ }^{141}$.

It should be noted, however, that transcriptional changes in exosome mutants can often be indirect. In S. pombe, heterochromatin is transcriptionally silenced through an RNAidependent mechanism that results in the deposition of the repressive chromatin mark histone 3 Lys 9 trimethylation (H3K9me3). In rrp6 mutants, H3K9me3 deposition is deregulated, which leads to reduced silencing of constitutive heterochromatic regions such as centromeres and induces the formation of novel heterochromatin domains not present in wild-type cells. The altered pattern of heterochromatinization correlates with changes in the expression levels of small RNAs in the exosome mutant ${ }^{25,142-145}$. Equally, the exosome in Arabidopsis was found to control the expression of several small interfering RNAs (siRNAs). Following the mutation of the exosome core subunit RRP45b, but not of its paralogue RRP45a, specific siRNA species accumulated, leading to increased repression of their target genes ${ }^{146}$. The opposite scenario was described in humans: RRP6 mediates transcriptional silencing of the HIV-1 promoter in the absence of a viral transactivator by processing the viral TAR transcript into small RNAs, which were proposed to hybridize with the promoter and inhibit its activity. When RRP6 is mutated, the inhibition of the HIV-1 promoter is lost ${ }^{147}$. Other examples in which the exosome modulates small RNA production have been reported, and overall, the scope of indirect regulation of mRNA levels by the exosome is potentially vast.

DNA damage repair and genomic integrity. The DDR is one of many cellular processes to which changes in post-transcriptional regulation of gene expression are known to contribute $^{148}$. Thus, it may not be too surprising that the activity of the nuclear exosome is regulated in response to DNA damage. RNA-binding protein 7 (RBM7), for example, a component of the human nuclear exosome targeting complex NEXT, is phosphorylated following UV-induced DNA damage. This leads to its binding by the adaptor protein 14-3-3 
and reduces its association with exosome substrates such as PROMPTs, possibly freeing it to pursue DNA damage-related tasks ${ }^{149}$. Furthermore, a direct involvement of exosome factors in the repair of DNA lesions has also been suggested. The nuclear matrix protein Rrp47 (C1D in humans), which forms a stable heterodimer with Rrp6 $6^{150,151}$, is required for the repair of UV-induced lesions in S. cerevisiae ${ }^{152,153}$. Human C1D was found to bind the Translinassociated factor (TRAX) and to activate the DNA-dependent protein kinase (DNA-PK), both known to play a role in DSB repair ${ }^{154,155}$. In Drosophila melanogaster, DSB repair is also compromised in the absence of $r r p 6^{156}$. Intriguingly, it was recently reported that DSBs can be repaired by an RNA-template-based mechanism that involves the formation of RNA-DNA hybrids at the break points ${ }^{157}$, and it is conceivable that the exosome is required to remove the RNA template to allow DNA repair to proceed.

RNA-DNA hybrids also occur as a by-product of transcription. Unless removed, the resulting R-loop structure can lead to DSBs ${ }^{158,159}$ (Box 1). It has been suggested repeatedly that the exosome could play a role in the resolution of R-loops and thereby contribute to genome integrity ${ }^{9,19,160,161}$. Strikingly, the increased incidence of genomic rearrangements in S. cerevisiae exosome mutants can be rescued by overexpression of RNase $\mathrm{H}$, which removes RNA-DNA hybrids ${ }^{162}$. In mouse, loci that produce exosome-targeted transcripts were found to accumulate R-loops following exosome knockdown ${ }^{9,19}$. Moreover, in human cells the exosome is recruited to R-loops that form at sites of replication-transcription collision ${ }^{160}$. In this case, exosome recruitment depended on the induction of a SUMO-dependent interaction between the R-loop-associated helicase Senataxin (SETX) ${ }^{163,164}$ and the exosome subunit RRP45. Recessive SETX mutations that prevent its association with RRP45 cause ataxia with oculomotor apraxia 2 (AOA2), one of several human Mendelian diseases that have been functionally linked to the exosome complex ${ }^{160}$. 
Antibody class switch recombination in B cells. The nuclear exosome has been implicated in another biological process that is intimately linked with R-loops: the diversification of immunoglobulins by somatic hypermutation and antibody class switch recombination (CSR) in B lymphocytes ${ }^{165}$. CSR is initiated by activation-induced cytidine deaminase (AID), which induces DSBs by modifying both DNA strands at the class switch region. The ensuing repair by homologous recombination results in rearrangements of the antibody locus and the production of different antibody isotypes. In mouse, it was proposed that recruitment of the exosome to the class switch region, which is G-rich and very prone to R-loop formation ${ }^{166}$, is required for optimal $\mathrm{CSR}^{161}$. If the exosome is mutated, the DNA strand involved in DNARNA hybrid formation across the class switch region is protected from deamination, again suggesting that the exosome may help to resolve R-loops ${ }^{161}$. Interestingly, AID frequently associates with other loci that produce unstable RNAs targeted by the exosome ${ }^{9,165}$. In exosome mutants, these loci accumulate R-loops. Mis-targeting AID to other loci is dangerous, and can cause genomic translocations that are associated with cancer ${ }^{165}$. A model how the RNA molecule in the RNA-DNA hybrid could be rendered susceptible to the exosome has also been suggested (Figure 4): In human B cells, Pol II bound by AID was found to be ubiquitylated by the E3 ubiquitin ligase Nedd $4^{167}$, the same enzyme that targets Pol II in response to UV damage, thereby inducing degradation of Pol II by the proteasome $^{168}$. In the absence of NEDD4, exosome RNA substrates at AID target genes were stabilized, and it was suggested that ubiquitin-dependent destabilization of Pol II stalled in an R-loop is required to expose RNA 3'OH ends, which are substrates for the exosome (Figure 4$)^{167}$. It has also been hypothesized that, alternatively, backtracking of an R-loopproducing Pol II could provide a means of exposing the required 3’OH end ${ }^{165}$. As was suggested for $S$. pombe $e^{138}$, this could allow the exosome to disassociate stalled Pol II and resolve the associated R-loop ${ }^{165}$. 


\section{Concluding remarks}

Recent years have seen a considerable advance in our understanding of how the nuclear exosome is regulated. Nevertheless, central aspects of substrate recognition still remain obscure, particularly in organisms other than S. cerevisiae, and the quest for factors that determine RNA fates - stable expression or rapid degradation - is still on. We have begun to understand that changes in RNA processing coupled to exosome-dependent decay constitute an important means of global gene regulation and expect to see the identification of many more genetic programmes regulated by the RNA exosome. Up to now, research has focused on the regulation of splicing as the primary mechanism of exosome-mediated gene regulation, but other RNA maturation processes, such as 3' end formation, may turn out to be as heavily exploited for regulatory purposes. RNA-DNA hybrids appear to be universal byproducts of transcription and are increasingly linked to disease $\mathrm{s}^{158,159,169,170}$. Thus, the recent work that links the exosome to R-loop dynamics is likely to have important implications, because it directly connects RNA surveillance with transcription regulation and may represent a general mechanism of exosome targeting. The data on R-loops discussed in this review is suggestive, and we are looking forward to future work that will corroborate it.

\section{References}

1. Chlebowski, A., Lubas, M., Jensen, T. H. \& Dziembowski, A. RNA decay machines: the exosome. Biochim. Biophys. Acta 1829, 552-60 (2013).

2. Mitchell, P. Exosome substrate targeting: the long and short of it. Biochem. Soc. Trans. 42, 1129-34 (2014).

3. Mitchell, P., Petfalski, E., Shevchenko, A., Mann, M. \& Tollervey, D. The Exosome: 
A Conserved Eukaryotic RNA Processing Complex Containing Multiple $3{ }^{\prime} \rightarrow 5^{\prime}$

Exoribonucleases. Cell 91, 457-466 (1997).

In this study the exosome complex was originally described and identifed as important for $5.8 \mathrm{~S}$ rRNA processing.

4. Allmang, C. et al. Functions of the exosome in rRNA, snoRNA and snRNA synthesis. EMBO J. 18, 5399-410 (1999).

5. Kiss, D. L. \& Andrulis, E. D. Genome-wide analysis reveals distinct substrate specificities of Rrp6, Dis3, and core exosome subunits. RNA 16, 781-91 (2010).

6. Chekanova, J. A. et al. Genome-wide high-resolution mapping of exosome substrates reveals hidden features in the Arabidopsis transcriptome. Cell 131, 1340-53 (2007).

7. Schneider, C., Kudla, G., Wlotzka, W., Tuck, A. C. \& Tollervey, D. Transcriptomewide analysis of exosome targets. Mol. Cell 48, 422-33 (2012).

8. Gudipati, R. K. et al. Extensive degradation of RNA precursors by the exosome in wild-type cells. Mol. Cell 48, 409-21 (2012).

9. Pefanis, E. et al. Noncoding RNA transcription targets AID to divergently transcribed loci in B cells. Nature 514, 389-93 (2014).

10. Szczepinska, T. et al. DIS3 shapes the RNA polymerase II transcriptome in humans by degrading a variety of unwanted transcripts. Genome Res. 25, 1622-33 (2015).

References 5 to 10 describe genome-wide analyses of exosome targets in different species.

11. Ntini, E. et al. Polyadenylation site-induced decay of upstream transcripts enforces promoter directionality. Nat. Struct. Mol. Biol. 20, 923-8 (2013).

12. Neil, H. et al. Widespread bidirectional promoters are the major source of cryptic transcripts in yeast. Nature 457, 1038-42 (2009).

13. $\mathrm{Xu}, \mathrm{Z}$. et al. Bidirectional promoters generate pervasive transcription in yeast. Nature 457, 1033-7 (2009).

14. Wyers, F. et al. Cryptic pol II transcripts are degraded by a nuclear quality control pathway involving a new poly(A) polymerase. Cell 121, 725-37 (2005).

The first study to show that CUTs in S. cerevisiae are degraded by the exosome complex in a TRAMP-dependent manner. 
15. Davis, C. A. \& Ares, M. Accumulation of unstable promoter-associated transcripts upon loss of the nuclear exosome subunit Rrp6p in Saccharomyces cerevisiae. Proc. Natl. Acad. Sci. U. S. A. 103, 3262-7 (2006).

16. Creamer, T. J. et al. Transcriptome-wide binding sites for components of the Saccharomyces cerevisiae non-poly(A) termination pathway: Nrd1, Nab3, and Sen1. PLoS Genet. 7, e1002329 (2011).

17. Wlotzka, W., Kudla, G., Granneman, S. \& Tollervey, D. The nuclear RNA polymerase II surveillance system targets polymerase III transcripts. EMBO J. 30, 1790-803 (2011).

18. Lubas, M. et al. Interaction profiling identifies the human nuclear exosome targeting complex. Mol. Cell 43, 624-37 (2011).

Identifies the first nuclear exosome activating complex in humans.

19. Pefanis, E. et al. RNA Exosome-Regulated Long Non-Coding RNA Transcription Controls Super-Enhancer Activity. Cell 161, 774-789 (2015).

20. Preker, P. et al. RNA exosome depletion reveals transcription upstream of active human promoters. Science 322, 1851-4 (2008).

21. Wang, S.-W., Stevenson, A. L., Kearsey, S. E., Watt, S. \& Bähler, J. Global role for polyadenylation-assisted nuclear RNA degradation in posttranscriptional gene silencing. Mol. Cell. Biol. 28, 656-65 (2008).

22. Flynn, R. A., Almada, A. E., Zamudio, J. R. \& Sharp, P. A. Antisense RNA polymerase II divergent transcripts are P-TEFb dependent and substrates for the RNA exosome. Proc. Natl. Acad. Sci. U. S. A. 108, 10460-5 (2011).

23. Houseley, J., Kotovic, K. M., El Hage, A. \& Tollervey, D. Trf4 targets ncRNAs from telomeric and rDNA spacer regions and functions in rDNA copy number control. EMBO J. 26, 4996-5006 (2007).

24. Vasiljeva, L., Kim, M., Terzi, N., Soares, L. M. \& Buratowski, S. Transcription termination and RNA degradation contribute to silencing of RNA polymerase II transcription within heterochromatin. Mol. Cell 29, 313-23 (2008).

25. Bühler, M., Haas, W., Gygi, S. P. \& Moazed, D. RNAi-dependent and -independent RNA turnover mechanisms contribute to heterochromatic gene silencing. Cell 129, 707-21 (2007).

Shows for the first time that the exosome complex plays an important role in posttranscriptional gene silencing at heterochromatic regions in S. pombe. 
26. Kadaba, S., Krueger, A. \& Trice, T. Nuclear surveillance and degradation of hypomodified initiator tRNAMet in S. cerevisiae. Genes Dev. 18, 1227-1240 (2004).

27. Bitton, D. A. et al. Widespread exon-skipping triggers degradation by nuclear RNA surveillance in fission yeast. Genome Res. 25, 884-96 (2015).

28. Bousquet-Antonelli, C., Presutti, C. \& Tollervey, D. Identification of a regulated pathway for nuclear pre-mRNA turnover. Cell 102, 765-75 (2000).

29. Coy, S., Volanakis, A., Shah, S. \& Vasiljeva, L. The Sm complex is required for the processing of non-coding RNAs by the exosome. PLoS One 8, e65606 (2013).

30. Eckard, S. C. et al. The SKIV2L RNA exosome limits activation of the RIG-I-like receptors. Nat. Immunol. 15, 839-45 (2014).

31. Wahba, L., Gore, S. K. \& Koshland, D. The homologous recombination machinery modulates the formation of RNA-DNA hybrids and associated chromosome instability. Elife 2, e00505 (2013).

32. Harigaya, Y. et al. Selective elimination of messenger RNA prevents an incidence of untimely meiosis. Nature 442, 45-50 (2006).

Identifies Mmi1 as an exosome specificity factor that targets mRNAs in $S$. pombe.

33. Yap, K., Lim, Z. Q., Khandelia, P., Friedman, B. \& Makeyev, E. V. Coordinated regulation of neuronal mRNA steady-state levels through developmentally controlled intron retention. Genes Dev. 26, 1209-23 (2012).

Introduces the idea that regulated suppression of splicing coupled to pre-mRNA decay by the exosome complex is employed to regulate gene expression.

34. Shah, S., Wittmann, S., Kilchert, C. \& Vasiljeva, L. IncRNA recruits RNAi and the exosome to dynamically regulate pho1 expression in response to phosphate levels in fission yeast. Genes Dev. 28, 231-44 (2014).

35. Kilchert, C. et al. Regulation of mRNA levels by decay-promoting introns that recruit the exosome specificity factor Mmi1. Cell Rep. 13, 1-12 (2015).

36. Hou, D., Ruiz, M. \& Andrulis, E. D. The ribonuclease Dis3 is an essential regulator of the developmental transcriptome. BMC Genomics 13, 359 (2012).

37. Wan, J. et al. Mutations in the RNA exosome component gene EXOSC3 cause pontocerebellar hypoplasia and spinal motor neuron degeneration. Nat. Genet. 44, 
704-8 (2012).

38. Boczonadi, V. et al. EXOSC8 mutations alter mRNA metabolism and cause hypomyelination with spinal muscular atrophy and cerebellar hypoplasia. Nat. Commun. 5, 4287 (2014).

39. Januszyk, K. \& Lima, C. D. The eukaryotic RNA exosome. Curr. Opin. Struct. Biol. 24, 132-40 (2014).

40. Tomecki, R. et al. The human core exosome interacts with differentially localized processive RNases: hDIS3 and hDIS3L. EMBO J. 29, 2342-57 (2010).

41. Makino, D. L., Baumgärtner, M. \& Conti, E. Crystal structure of an RNA-bound 11subunit eukaryotic exosome complex. Nature 495, 70-5 (2013).

42. Drazkowska, K. et al. The RNA exosome complex central channel controls both exonuclease and endonuclease Dis3 activities in vivo and in vitro. Nucleic Acids Res. 41, 3845-58 (2013).

43. Liu, J.-J. et al. Visualization of distinct substrate-recruitment pathways in the yeast exosome by EM. Nat. Struct. Mol. Biol. 21, 95-102 (2014).

44. Wasmuth, E. V, Januszyk, K. \& Lima, C. D. Structure of an Rrp6-RNA exosome complex bound to poly(A) RNA. Nature 511, 435-9 (2014).

First structure of the RNA-bound core associated with Rrp6. Suggests entry of the RNA molecule from the side to reach the active site of Rrp6.

45. Lebreton, A. \& Séraphin, B. Exosome-mediated quality control: substrate recruitment and molecular activity. Biochim. Biophys. Acta 1779, 558-65 (2008).

46. Schaeffer, D. et al. The exosome contains domains with specific endoribonuclease, exoribonuclease and cytoplasmic mRNA decay activities. Nat. Struct. Mol. Biol. 16, 56-62 (2009).

47. Makino, D. L. et al. RNA degradation paths in a 12-subunit nuclear exosome complex. Nature 524, 54-8 (2015).

Presents a crystal structure of an RNA-bound exosome complex that includes Dis3 and Rrp6. Suggest that RNA can reach Rrp6 without making contact with other units of the complex.

48. Wasmuth, E. V \& Lima, C. D. Exo- and endoribonucleolytic activities of yeast cytoplasmic and nuclear RNA exosomes are dependent on the noncatalytic core and 
central channel. Mol. Cell 48, 133-44 (2012).

49. Lykke-Andersen, S., Tomecki, R., Jensen, T. H. \& Dziembowski, A. The eukaryotic RNA exosome: Same scaffold but variable catalytic subunits. RNA Biol. 8, 61-66 (2011).

50. Dziembowski, A., Lorentzen, E., Conti, E. \& Séraphin, B. A single subunit, Dis3, is essentially responsible for yeast exosome core activity. Nat. Struct. Mol. Biol. 14, 1522 (2007).

51. Liu, Q., Greimann, J. C. \& Lima, C. D. Reconstitution, activities, and structure of the eukaryotic RNA exosome. Cell 127, 1223-37 (2006).

References 50 and 51 provide biochemical characterization of the RNA-degrading activities of the exosome complex.

52. Bonneau, F., Basquin, J., Ebert, J., Lorentzen, E. \& Conti, E. The yeast exosome functions as a macromolecular cage to channel RNA substrates for degradation. Cell 139, 547-59 (2009).

53. Hardwick, S. W. \& Luisi, B. F. Rarely at rest: RNA helicases and their busy contributions to RNA degradation, regulation and quality control. RNA Biol. 10, 56-70 (2013).

54. Halbach, F., Reichelt, P., Rode, M. \& Conti, E. The yeast ski complex: crystal structure and RNA channeling to the exosome complex. Cell 154, 814-26 (2013).

55. LaCava, J. et al. RNA degradation by the exosome is promoted by a nuclear polyadenylation complex. Cell 121, 713-24 (2005).

56. Jia, H. et al. The RNA helicase Mtr4p modulates polyadenylation in the TRAMP complex. Cell 145, 890-901 (2011).

57. Lubas, M. et al. The Human Nuclear Exosome Targeting Complex Is Loaded onto Newly Synthesized RNA to Direct Early Ribonucleolysis. Cell Rep. 10, 178-92 (2015).

58. Hrossova, D. et al. RBM7 subunit of the NEXT complex binds U-rich sequences and targets 3'-end extended forms of snRNAs. Nucleic Acids Res. 43, 4236-48 (2015).

59. Win, T. Z. et al. Requirement of fission yeast Cid14 in polyadenylation of rRNAs. Mol. Cell. Biol. 26, 1710-21 (2006). 
60. Lee, N. N. et al. Mtr4-like protein coordinates nuclear RNA processing for heterochromatin assembly and for telomere maintenance. Cell 155, 1061-74 (2013).

61. Zhou, Y. et al. The fission yeast MTREC complex targets CUTs and unspliced premRNAs to the nuclear exosome. Nat. Commun. 6, 7050 (2015).

62. Egan, E. D., Braun, C. R., Gygi, S. P. \& Moazed, D. Post-transcriptional regulation of meiotic genes by a nuclear RNA silencing complex. RNA 20, 867-81 (2014).

63. Lange, H. et al. The RNA helicases AtMTR4 and HEN2 target specific subsets of nuclear transcripts for degradation by the nuclear exosome in Arabidopsis thaliana. PLoS Genet. 10, e1004564 (2014).

64. Weir, J. R., Bonneau, F., Hentschel, J. \& Conti, E. Structural analysis reveals the characteristic features of Mtr4, a DExH helicase involved in nuclear RNA processing and surveillance. Proc. Natl. Acad. Sci. U. S. A. 107, 12139-44 (2010).

65. Halbach, F., Rode, M. \& Conti, E. The crystal structure of S. cerevisiae Ski2, a DExH helicase associated with the cytoplasmic functions of the exosome. RNA 18, 124-34 (2012).

66. Chen, C. Y. et al. AU binding proteins recruit the exosome to degrade ARE-containing mRNAs. Cell 107, 451-64 (2001).

67. Tran, H., Schilling, M., Wirbelauer, C., Hess, D. \& Nagamine, Y. Facilitation of mRNA deadenylation and decay by the exosome-bound, DExH protein RHAU. Mol. Cell 13, 101-11 (2004).

68. Thoms, M. et al. The Exosome Is Recruited to RNA Substrates through Specific Adaptor Proteins. Cell 162, 1029-1038 (2015).

Identifies exosome-specificity factors involved in rRNA processing in S. cerevisiae.

69. Steinmetz, E. J., Conrad, N. K., Brow, D. a \& Corden, J. L. RNA-binding protein Nrd1 directs poly(A)-independent 3'-end formation of RNA polymerase II transcripts. Nature 413, 327-31 (2001).

70. Schulz, D. et al. Transcriptome Surveillance by Selective Termination of Noncoding RNA Synthesis. Cell 155, 1075-1087 (2013).

71. Kim, M. et al. Distinct pathways for snoRNA and mRNA termination. Mol. Cell 24, 723-34 (2006). 
72. Vasiljeva, L., Kim, M., Mutschler, H., Buratowski, S. \& Meinhart, A. The Nrd1-Nab3Sen1 termination complex interacts with the Ser5-phosphorylated RNA polymerase II C-terminal domain. Nat. Struct. Mol. Biol. 15, 795-804 (2008).

73. Tudek, A. et al. Molecular basis for coordinating transcription termination with noncoding RNA degradation. Mol. Cell 55, 467-81 (2014).

74. Vasiljeva, L. \& Buratowski, S. Nrd1 interacts with the nuclear exosome for 3' processing of RNA polymerase II transcripts. Mol. Cell 21, 239-48 (2006).

References 73 and 74 describe how the exosome specificity factor Nrd1 recruits TRAMP and the exosome complex to degrade NNS-terminated RNAs.

75. Porrua, O. \& Libri, D. Transcription termination and the control of the transcriptome: why, where and how to stop. Nat. Rev. Mol. Cell Biol. 16, 190-202 (2015).

76. Preker, P. et al. PROMoter uPstream Transcripts share characteristics with mRNAs and are produced upstream of all three major types of mammalian promoters. Nucleic Acids Res. 39, 7179-93 (2011).

77. Nojima, T. et al. Mammalian NET-Seq Reveals Genome-wide Nascent Transcription Coupled to RNA Processing. Cell 161, 526-540 (2015).

78. Hiriart, E. et al. Mmi1 RNA surveillance machinery directs RNAi complex RITS to specific meiotic genes in fission yeast. EMBO J. 31, 2296-308 (2012).

79. Yamanaka, S., Yamashita, A., Harigaya, Y., Iwata, R. \& Yamamoto, M. Importance of polyadenylation in the selective elimination of meiotic mRNAs in growing $\mathrm{S}$. pombe cells. EMBO J. 29, 2173-81 (2010).

80. St-André, O. et al. Negative regulation of meiotic gene expression by the nuclear poly(a)-binding protein in fission yeast. J. Biol. Chem. 285, 27859-68 (2010).

81. Ard, R., Tong, P. \& Allshire, R. C. Long non-coding RNA-mediated transcriptional interference of a permease gene confers drug tolerance in fission yeast. Nat. Commun. 5, 5576 (2014).

82. Yamashita, A. et al. Hexanucleotide motifs mediate recruitment of the RNA elimination machinery to silent meiotic genes. Open Biol. 2, 120014 (2012).

83. Wang, X. et al. N6-methyladenosine-dependent regulation of messenger RNA stability. Nature 505, 117-20 (2014). 
84. Zhu, T. et al. Crystal structure of the YTH domain of YTHDF2 reveals mechanism for recognition of N6-methyladenosine. Cell Res. 24, 1493-6 (2014).

85. Carroll, K. L., Ghirlando, R., Ames, J. M. \& Corden, J. L. Interaction of yeast RNAbinding proteins Nrd1 and Nab3 with RNA polymerase II terminator elements. RNA 13, 361-73 (2007).

86. Grenier St-Sauveur, V., Soucek, S., Corbett, A. H. \& Bachand, F. Poly(A) tailmediated gene regulation by opposing roles of Nab2 and Pab2 nuclear poly(A)-binding proteins in pre-mRNA decay. Mol. Cell. Biol. 33, 4718-31 (2013).

87. Schmid, M. et al. The Nuclear PolyA-Binding Protein Nab2p Is Essential for mRNA Production. Cell Rep. 12, 128-39 (2015).

88. van Hoof, A., Lennertz, P. \& Parker, R. Yeast Exosome Mutants Accumulate 3'Extended Polyadenylated Forms of U4 Small Nuclear RNA and Small Nucleolar RNAs. Mol. Cell. Biol. 20, 441-452 (2000).

89. Mitchell, J. R., Cheng, J. \& Collins, K. A box H/ACA small nucleolar RNA-like domain at the human telomerase RNA 3' end. Mol. Cell. Biol. 19, 567-76 (1999).

90. Yin, Q.-F. et al. Long noncoding RNAs with snoRNA ends. Mol. Cell 48, 219-30 (2012).

91. Wilusz, J. E. Long noncoding RNAs: Re-writing dogmas of RNA processing and stability. Biochim. Biophys. Acta (2015). doi:10.1016/j.bbagrm.2015.06.003

92. Tuck, A. C. \& Tollervey, D. A transcriptome-wide atlas of RNP composition reveals diverse classes of mRNAs and lncRNAs. Cell 154, 996-1009 (2013).

93. Andersson, R. et al. Nuclear stability and transcriptional directionality separate functionally distinct RNA species. Nat. Commun. 5, 5336 (2014).

References 92 and 93 confirm that susceptibility to degradation by the exosome complex is a global determinant of RNA fate in the nucleus.

94. Colin, J. et al. Roadblock termination by reb1p restricts cryptic and readthrough transcription. Mol. Cell 56, 667-80 (2014).

95. Rondón, A. G., Mischo, H. E., Kawauchi, J. \& Proudfoot, N. J. Fail-safe transcriptional termination for protein-coding genes in S. cerevisiae. Mol. Cell 36, 8898 (2009). 
96. Gudipati, R. K., Neil, H., Feuerbach, F., Malabat, C. \& Jacquier, A. The yeast RPL9B gene is regulated by modulation between two modes of transcription termination.

EMBO J. 31, 2427-37 (2012).

97. Webb, S., Hector, R. D., Kudla, G. \& Granneman, S. PAR-CLIP data indicate that Nrd1-Nab3-dependent transcription termination regulates expression of hundreds of protein coding genes in yeast. Genome Biol. 15, R8 (2014).

98. Gullerova, M. \& Proudfoot, N. J. Transcriptional interference and gene orientation in yeast: noncoding RNA connections. Cold Spring Harb. Symp. Quant. Biol. 75, 299311 (2010).

99. Lemieux, C. et al. A Pre-mRNA degradation pathway that selectively targets introncontaining genes requires the nuclear poly(A)-binding protein. Mol. Cell 44, 108-19 (2011).

100. Bergeron, D., Pal, G., Beaulieu, Y. B., Chabot, B. \& Bachand, F. Regulated intron retention and nuclear pre-mRNA decay contribute to PABPN1 autoregulation. Mol. Cell. Biol. 35, 2503-17 (2015).

101. Muniz, L., Davidson, L. \& West, S. Poly(A) polymerase and the nuclear poly(A) binding protein, PABPN1, coordinate the splicing and degradation of a subset of human pre-mRNAs. Mol. Cell. Biol. 35, 2218-30 (2015).

102. Schmid, M. et al. Rrp6p controls mRNA poly(A) tail length and its decoration with poly(A) binding proteins. Mol. Cell 47, 267-80 (2012).

103. Bresson, S. M. \& Conrad, N. K. The human nuclear poly(a)-binding protein promotes RNA hyperadenylation and decay. PLoS Genet. 9, e1003893 (2013).

104. Hackmann, A. et al. Quality control of spliced mRNAs requires the shuttling SR proteins Gbp2 and Hrb1. Nat. Commun. 5, 3123 (2014).

105. Alexander, R. D. \& Beggs, J. D. Cross-talk in transcription, splicing and chromatin: who makes the first call? Biochem. Soc. Trans. 38, 1251-6 (2010).

106. Kong, K.-Y. E. et al. Cotranscriptional recruitment of yeast TRAMP complex to intronic sequences promotes optimal pre-mRNA splicing. Nucleic Acids Res. 42, 64360 (2014).

107. Nag, A. \& Steitz, J. Tri-snRNP-associated proteins interact with subunits of the TRAMP and nuclear exosome complexes, linking RNA decay and pre-mRNA 
splicing. RNA Biol. 9, 334-42 (2012).

108. Libri, D. et al. Interactions between mRNA Export Commitment, 3'-End Quality Control, and Nuclear Degradation. Mol. Cell. Biol. 22, 8254-8266 (2002).

109. Rougemaille, M. et al. Dissecting mechanisms of nuclear mRNA surveillance in THO/sub2 complex mutants. EMBO J. 26, 2317-26 (2007).

110. Saguez, C. et al. Nuclear mRNA surveillance in THO/sub2 mutants is triggered by inefficient polyadenylation. Mol. Cell 31, 91-103 (2008).

111. de Almeida, S. F., García-Sacristán, A., Custódio, N. \& Carmo-Fonseca, M. A link between nuclear RNA surveillance, the human exosome and RNA polymerase II transcriptional termination. Nuc. Ac. Res. 38, 8015-26 (2010).

112. Eberle, A. B. et al. Splice-site mutations cause Rrp6-mediated nuclear retention of the unspliced RNAs and transcriptional down-regulation of the splicing-defective genes. PLoS One 5, e11540 (2010).

113. Hilleren, P., McCarthy, T., Rosbash, M., Parker, R. \& Jensen, T. H. Quality control of mRNA 3'-end processing is linked to the nuclear exosome. Nature 413, 538-42 (2001).

Shows the requirement for Rrp6 in the retention of aberrant transcripts at the site of transcription.

114. Custódio, N. et al. Inefficient processing impairs release of RNA from the site of transcription. EMBO J. 18, 2855-66 (1999).

115. Kallehauge, T. B., Robert, M.-C., Bertrand, E. \& Jensen, T. H. Nuclear retention prevents premature cytoplasmic appearance of mRNA. Mol. Cell 48, 145-52 (2012).

116. Keller, C. \& Bühler, M. Chromatin-associated ncRNA activities. Chromosome Res. 21, 627-41 (2013).

117. Zhang, H. et al. An Rrp6-like protein positively regulates noncoding RNA levels and DNA methylation in Arabidopsis. Mol. Cell 54, 418-30 (2014).

Demonstrates a biological function for the Rrp6-dependent retention of a regulatory ncRNA at the chromatin.

118. de Nadal, E., Ammerer, G. \& Posas, F. Controlling gene expression in response to stress. Nat. Rev. Genet. 12, 833-45 (2011). 
119. Reis, C. C. \& Campbell, J. L. Contribution of Trf4/5 and the nuclear exosome to genome stability through regulation of histone mRNA levels in Saccharomyces cerevisiae. Genetics 175, 993-1010 (2007).

120. Canavan, R. \& Bond, U. Deletion of the nuclear exosome component RRP6 leads to continued accumulation of the histone mRNA HTB1 in S-phase of the cell cycle in Saccharomyces cerevisiae. Nucleic Acids Res. 35, 6268-79 (2007).

121. Arigo, J. T., Carroll, K. L., Ames, J. M. \& Corden, J. L. Regulation of yeast NRD1 expression by premature transcription termination. Mol. Cell 21, 641-51 (2006).

122. Kim, K.-Y. \& Levin, D. E. Mpk1 MAPK association with the Paf1 complex blocks Sen1-mediated premature transcription termination. Cell 144, 745-56 (2011).

123. Volanakis, A. et al. Spliceosome-mediated decay (SMD) regulates expression of nonintronic genes in budding yeast. Genes Dev. 27, 2025-2038 (2013).

124. Roy, K. \& Chanfreau, G. Stress-induced nuclear RNA degradation pathways regulate yeast bromodomain factor 2 to promote cell survival. PLoS Genet. 10, e1004661 (2014).

125. Barta, I. \& Iggo, R. D. Autoregulation of expression of the yeast Dbp2p 'DEAD-box' protein is mediated by sequences in the conserved DBP2 intron. EMBO J. 14, 3800-8 (1995).

126. Preker, P. J. \& Guthrie, C. Autoregulation of the mRNA export factor Yra1p requires inefficient splicing of its pre-mRNA. RNA 12, 994-1006 (2006).

127. Roth, K. M., Byam, J., Fang, F. \& Butler, J. S. Regulation of NAB2 mRNA 3'-end formation requires the core exosome and the Trf4p component of the TRAMP complex. RNA 15, 1045-58 (2009).

128. Macías, S., Bragulat, M., Tardiff, D. F. \& Vilardell, J. L30 binds the nascent RPL30 transcript to repress U2 snRNP recruitment. Mol. Cell 30, 732-42 (2008).

129. Plocik, A. M. \& Guthrie, C. Diverse forms of RPS9 splicing are part of an evolving autoregulatory circuit. PLoS Genet. 8, e1002620 (2012).

130. Malygin, A. A., Parakhnevitch, N. M., Ivanov, A. V, Eperon, I. C. \& Karpova, G. G. Human ribosomal protein S13 regulates expression of its own gene at the splicing step by a feedback mechanism. Nucleic Acids Res. 35, 6414-23 (2007). 
131. Colak, D., Ji, S.-J., Porse, B. T. \& Jaffrey, S. R. Regulation of axon guidance by compartmentalized nonsense-mediated mRNA decay. Cell 153, 1252-65 (2013).

132. Wong, J. J.-L. et al. Orchestrated intron retention regulates normal granulocyte differentiation. Cell 154, 583-95 (2013).

133. Braunschweig, U. et al. Widespread intron retention in mammals functionally tunes transcriptomes. Genome Res. 24, 1774-86 (2014).

134. Parenteau, J. et al. Introns within ribosomal protein genes regulate the production and function of yeast ribosomes. Cell 147, 320-31 (2011).

135. Danin-Kreiselman, M., Lee, C. Y. \& Chanfreau, G. RNAse III-Mediated Degradation of Unspliced Pre-mRNAs and Lariat Introns. Mol. Cell 11, 1279-1289 (2003).

136. Castelnuovo, M. et al. Bimodal expression of PHO84 is modulated by early termination of antisense transcription. Nat. Struct. Mol. Biol. 20, 851-8 (2013).

137. Fox, M. J., Gao, H., Smith-Kinnaman, W. R., Liu, Y. \& Mosley, A. L. The exosome component Rrp6 is required for RNA polymerase II termination at specific targets of the Nrd1-Nab3 pathway. PLoS Genet. 11, e1004999 (2015).

138. Lemay, J.-F. et al. The RNA exosome promotes transcription termination of backtracked RNA polymerase II. Nat. Struct. Mol. Biol. 21, 919-26 (2014).

Suggests that the exosome complex helps to disassociate stalled Pol II complexes from the DNA.

139. Kim, M. et al. The yeast Rat1 exonuclease promotes transcription termination by RNA polymerase II. Nature 432, 517-22 (2004).

140. West, S., Gromak, N. \& Proudfoot, N. J. Human 5' $\rightarrow$ 3' exonuclease Xrn2 promotes transcription termination at co-transcriptional cleavage sites. Nature 432, 522-525 (2004).

141. Skourti-Stathaki, K., Kamieniarz-Gdula, K. \& Proudfoot, N. J. R-loops induce repressive chromatin marks over mammalian gene terminators. Nature 516, 436-9 (2014).

142. Zhang, K. et al. Clr4/Suv39 and RNA quality control factors cooperate to trigger RNAi and suppress antisense RNA. Science 331, 1624-1627 (2011).

143. Yamanaka, S. et al. RNAi triggered by specialized machinery silences developmental 
genes and retrotransposons. Nature 493, 557-60 (2013).

144. Bühler, M., Spies, N., Bartel, D. P. \& Moazed, D. TRAMP-mediated RNA surveillance prevents spurious entry of RNAs into the Schizosaccharomyces pombe siRNA pathway. Nat. Struct. Mol. Biol. 15, 1015-23 (2008).

145. Coy, S. \& Vasiljeva, L. The exosome and heterochromatin : multilevel regulation of gene silencing. Adv. Exp. Med. Biol. 702, 105-21 (2011).

146. Lam, P. et al. The exosome and trans-acting small interfering RNAs regulate cuticular wax biosynthesis during Arabidopsis inflorescence stem development. Plant Physiol. 167, 323-36 (2015).

147. Wagschal, A. et al. Microprocessor, Setx, Xrn2, and Rrp6 co-operate to induce premature termination of transcription by RNAPII. Cell 150, 1147-57 (2012).

148. McKay, B. C. Post-transcriptional regulation of DNA damage-responsive gene expression. Antioxid. Redox Signal. 20, 640-54 (2014).

149. Blasius, M., Wagner, S. A., Choudhary, C., Bartek, J. \& Jackson, S. P. A quantitative 14-3-3 interaction screen connects the nuclear exosome targeting complex to the DNA damage response. Genes Dev. 28, 1977-82 (2014).

150. Mitchell, P. et al. Rrp47p is an exosome-associated protein required for the 3' processing of stable RNAs. Mol. Cell. Biol. 23, 6982-92 (2003).

151. Schuch, B. et al. The exosome-binding factors Rrp6 and Rrp47 form a composite surface for recruiting the Mtr4 helicase. EMBO J. 33, 2829-46 (2014).

152. Hieronymus, H., Yu, M. C. \& Silver, P. A. Genome-wide mRNA surveillance is coupled to mRNA export. Genes Dev. 18, 2652-62 (2004).

153. Erdemir, T., Bilican, B., Cagatay, T., Goding, C. R. \& Yavuzer, U. Saccharomyces cerevisiae C1D is implicated in both non-homologous DNA end joining and homologous recombination. Mol. Microbiol. 46, 947-57 (2002).

154. Erdemir, T., Bilican, B., Oncel, D., Goding, C. R. \& Yavuzer, U. DNA damagedependent interaction of the nuclear matrix protein C1D with Translin-associated factor X (TRAX). J. Cell Sci. 115, 207-16 (2002).

155. Yavuzer, U., Smith, G. C., Bliss, T., Werner, D. \& Jackson, S. P. DNA endindependent activation of DNA-PK mediated via association with the DNA-binding 
protein C1D. Genes Dev. 12, 2188-99 (1998).

156. Marin-Vicente, C., Domingo-Prim, J., Eberle, A. B. \& Visa, N. RRP6/EXOSC10 is required for the repair of DNA double-strand breaks by homologous recombination. $J$. Cell Sci. 128, 1097-107 (2015).

157. Keskin, H. et al. Transcript-RNA-templated DNA recombination and repair. Nature 515, 436-9 (2014).

158. Aguilera, A. \& García-Muse, T. R loops: from transcription byproducts to threats to genome stability. Mol. Cell 46, 115-24 (2012).

159. Sollier, J. \& Cimprich, K. A. Breaking bad: R-loops and genome integrity. Trends Cell Biol. 25, 514-522 (2015).

160. Richard, P., Feng, S. \& Manley, J. L. A SUMO-dependent interaction between Senataxin and the exosome, disrupted in the neurodegenerative disease AOA2, targets the exosome to sites of transcription-induced DNA damage. Genes Dev. 27, 2227-32 (2013).

First study to show a functional link between the repair of DNA damage, R-loop formation and the exosome complex.

161. Basu, U. et al. The RNA exosome targets the AID cytidine deaminase to both strands of transcribed duplex DNA substrates. Cell 144, 353-63 (2011).

162. Wahba, L., Amon, J. D., Koshland, D. \& Vuica-Ross, M. RNase H and multiple RNA biogenesis factors cooperate to prevent RNA:DNA hybrids from generating genome instability. Mol. Cell 44, 978-88 (2011).

163. Mischo, H. E. et al. Yeast Sen1 helicase protects the genome from transcriptionassociated instability. Mol. Cell 41, 21-32 (2011).

164. Skourti-Stathaki, K., Proudfoot, N. J. \& Gromak, N. Human senataxin resolves RNA/DNA hybrids formed at transcriptional pause sites to promote Xrn2-dependent termination. Mol. Cell 42, 794-805 (2011).

165. Pefanis, E. \& Basu, U. RNA Exosome Regulates AID DNA Mutator Activity in the B Cell Genome. Adv. Immunol. 127, 257-308 (2015).

166. Reaban, M. E. \& Griffin, J. A. Induction of RNA-stabilized DNA conformers by transcription of an immunoglobulin switch region. Nature 348, 342-4 (1990). 
167. Sun, J. et al. E3-ubiquitin ligase Nedd4 determines the fate of AID-associated RNA polymerase II in B cells. Genes Dev. 27, 1821-33 (2013).

168. Anindya, R., Aygün, O. \& Svejstrup, J. Q. Damage-induced ubiquitylation of human RNA polymerase II by the ubiquitin ligase Nedd4, but not Cockayne syndrome proteins or BRCA1. Mol. Cell 28, 386-97 (2007).

169. Santos-Pereira, J. M. \& Aguilera, A. R loops: new modulators of genome dynamics and function. Nat. Rev. Genet. 16, 583-97 (2015).

170. Groh, M. \& Gromak, N. Out of balance: R-loops in human disease. PLoS Genet. 10, e1004630 (2014).

\section{Acknowledgments}

The authors apologize to the many colleagues whose work could not be cited due to limited space. This work was supported by a research fellowship from the DFG (KI1657/1-1) to CK, a studentship by the MRC to SW and Research and Career and Senior Research fellowships from the Wellcome Trust to LV (WT088359MA and WT106994MA).

\section{Declaration of competing financial interests}

The authors declare no competing financial interests.

\section{Box 1: R-loops}

R-loops are RNA-DNA-hybrid structures that are by-product of transcription ${ }^{158,159}$. Sporadically, nascent RNA will invade the transcription bubble and hybridize with the template DNA strand, which leaves the non-template strand exposed as a single-stranded loop and can lead to polymerase stalling (see Figure 5). For a variety of reasons, R-loops 
frequently lead to DNA double strand breaks (DSBs), to such an extent that they contribute significantly to genomic instability ${ }^{158,159}$. Accordingly, cells employ various means to restrict R-loop formation. This includes both preventive measures, such as coating the nascent RNA with splicing or packaging factors such as the THO complex, as well as salvage pathways, such as hybrid unwinding by helicases such as Sen1 (SETX in humans) ${ }^{163,164}$ or the degradation of RNAs caught in R-loops by RNase $\mathrm{H}^{158,159}$. Recently, it was suggested that DNA-RNA hybrids can also form in trans, when RNA hybridizes with distant, homologous genomic sequences. RNA-DNA-hybrid formation in trans is mediated by the homologous recombination machinery and is integral to RNA template-mediated DSB repair ${ }^{31,157}$.

\section{Figure Legends}

Figure 1 | Structure of exosome complex isoforms. a | (Left) Model of the EXO11 ${ }^{\text {Dis3+Rrp6 }}$ complex. Surface structure of EXO11 ${ }^{\text {Dis3+Rrp6 }}$ was generated by superimposing the structures of EXO10 ${ }^{\text {Dis3+Rrp6Cterm }}$ (Rrp6 in red and the cap complex in green; PDB 4IFD) and EXO10 ${ }^{\mathrm{Rrp} 6}$ (PH ring complex in blue, Dis3 in purple and RNA in black; PDB 4OO1) from S. cerevisiae. (Right) Schematics of an RNA molecule threading 3' to 5' through the central channel of the cap and PH ring to the Dis3 exonucleolytic centre, where it is degraded. The arrows indicate the direction in which the RNA travels. b | There are two possible paths of RNA substrates to the Rrp6 active site when bound to the exosome complex. For route 1, the RNA enters Rrp6 as in (a), but instead of going into the central channel, it turns as it exits Rrp6 and reaches the active site with its 3' nucleotide. In this case, the RNA does not pass through the exosome core. Alternatively, as shown in route 2, the RNA enters the complex from the side, between the PH ring and S1/KH cap, and then bends upwards to reach the active site of Rrp6. Here, the RNA traverses the channel of the S1/KH cap with its 3' end first from bottom to top and 
therefore shows opposite orientation to (a), where the RNA enters from the top. $\mathbf{c}$ I The composition of exosome complexes in different compartments differs between yeast and human. In S. cerevisiae (left), exosome complexes with the Rrp6 subunit are exclusively localized in the nucleus, whereas exosomes with Dis3 can be found throughout the cell. In human cells (right), however, Dis3 is excluded from the nucleolus. The Dis3 homologue Dis3L also associates with the exosome core and is restricted to the cytoplasm.

Substoichiometric amounts of exosomes with different exonuclease subunits are present in the cytoplasm (indicated by relative sizes).

Figure 2 | Conserved features of RNA targeting to the exosome. a | Schematic model of the steps that lead to degradation of a generic exosome substrate. First, a binding site on the RNA is recognized by an exosome specificity factor (ESF). Substrate recognition may depend on RNA sequence, secondary structure and/or RNA modifications. The ESF then recruits an exosome activating complex that contains a conserved RNA helicase (substrate activation). Recruitment of the helicase can either occur directly or be mediated by accessory proteins (AP) contained within the complex. RNA unwinding renders the substrate susceptible to degradation by the exosome complex. b | Tightly regulated, short-lived human mRNAs frequently contain AU-rich elements (AREs), for example Urokinase-type (U)plasminogen activator mRNA or cytokine-encoding mRNAs. AREs are recognized by AREbinding proteins (ARE-BPs), which can recruit RNA helicases such as RHAU to enable subsequent degradation of the RNA by the exosome. c I Cryptic transcripts in S. cerevisiae are rapidly degraded by the exosome complex. The trimeric complex of Nrd1-Nab3-Sen1 (NNS) binds to nascent RNA that is enriched with specific sequence motifs. Nrd1 simultaneously interacts with phosphorylated Ser 5 in the heptad repeats of the C-terminal domain of Pol II. This modification is a hallmark of early elongating Pol II. Through an unknown mechanism, Nrd1 induces termination of Pol II transcription. Nrd1 is then free to 
bind Trf4 and to thus recruit the Mtr4-containing TRAMP complex to the RNA to unwind it and degrade it by the exosome complex. The NNS pathway is mostly restricted to short transcripts because it depends on the recognition of early elongating Pol II. d I During mitotic growth in $S$. pombe, meiotic mRNAs that are enriched with sequence motifs termed “determinants of selective removal” (DSRs), are rapidly degraded by the exosome complex. DSRs can be bound by the YTH protein Mmi1. Work on other YTH proteins suggests that DSR recognition could potentially involve N6-methylation of adenosines within the motif. Mmi1 recruits various factors, which are involved in regulating the stability of the transcript, including Red1, a constituent of the MTREC complex that contains the helicase Mtl1 (Table 1). RNA unwinding renders the substrate susceptible to degradation by the exosome complex. Mmi1 also targets several regulatory ncRNAs and pre-mRNAs.

Figure 3 | RNA surveillance by the exosome co-operates with RNA processing to regulate mRNA levels. a | RNA processing can lead to decay at different stages of mRNA biogenesis. Early during transcription, binding of the S. cerevisiae Nrd1-Nab3-Sen1 complex (NNS) to nascent mRNA can induce transcription termination coupled to decay by the exosome, and thus suppress gene expression (left). For example, the $N R D 1$ mRNA contains several Nrd1-binding sites, which are bound by the NNS complex when Nrd1 protein levels are high, thereby inducing premature transcription termination. When Nrd1 levels are low, full-length NRD1 mRNA can be produced. Similarly, mRNA transcribed from the stressresponsive gene FKS2 contains Nab3-binding sites and is terminated prematurely in normal growth conditions. Under conditions of cell wall stress, the physical association of a MAP kinase with the gene locus prevents recruitment of NNS and suppresses premature termination. Hence, more FKS2 mRNA is generated. Other mRNAs contain cryptic introns that need to be retained to produce functional transcript (right). During transcription elongation, these introns are generally spliced at low frequency. However, in response to 
environmental cues, splicing of cryptic introns can be activated, but remains non-productive. These splicing products are then rapidly degraded by the nuclear exosome in a process termed spliceosome-mediated decay (SMD). When levels of SMD are high, mRNA production is low. For example, splicing of a cryptic intron in the BDF2 mRNA is activated following DNA damage. $\mathbf{b}$ | Suppression of RNA processing can lead to decay at various stages of mRNA biogenesis. For example, recruitment of a splicing inhibitor (SI) can lead to intron retention. Pre-mRNAs with retained introns are rapidly degraded by the exosome complex. Known cases include the ribosomal protein genes rpl3002 and rps2202 in S. pombe. Here, it was proposed that the protein products of the paralogous genes, rpl3001 and rps2201, respectively, may bind to the retained introns and suppress splicing, thus reducing mRNA levels and controlling ribosomal protein homeostasis. During mouse neuronal development, expression of polypyrimidine tract-binding protein (Ptbp1) is decreased. Reduction of Ptbp1 levels alleviates the suppression of splicing and degradation by the exosome of several mRNAs that encode neuronal proteins, including Stxb1, which is a neuron-specific presynaptic protein. $\mathbf{c}$ | Schematic depicting the interplay between regulated RNA processing, exosomal degradation and mRNA levels for the example of intron retention. In conditions where splicing occurs rapidly, all nascent transcripts mature productively and a lot of mRNA is generated. When splicing is slow, a large fraction of transcripts is degraded, and little mRNA is produced. Through this mechanism, negative regulation of RNA processing can repress genes expression very efficiently.

Figure 4 | Potential mechanisms of R-loop resolution by the exosome complex. During transcription, nascent RNA can invade the transcription bubble and hybridize with the template DNA strand. This leaves the non-template strand exposed as a single-stranded loop and can lead to Pol II stalling. These R-loop structures can lead to DNA double strand breaks and indeed have been associated with genomic instability. The exosome has been suggested 
to play a role in the resolution of R-loops by degrading the RNA strand that forms the RNADNA hybrid. Two models of how the exosome could gain access to the RNA substrate have been proposed. First, stalled Pol II can be ubiquitylated by the E3 ligase Nedd4, which triggers its degradation by the proteasome, thus exposing a free 3’OH end. This could serve as a substrate for the exosome complex, which would then degrade the RNA and resolve the R-loop (left). R-loop resolution could be facilitated by additional factors, such as the helicase SETX (Sen1 in S. cerevisiae). Alternatively, the stalled polymerase is known to have a tendency to backtrack on the template strand, causing the 3’OH of the nascent transcript to protrude from the transcription complex (right). It has been suggested that this could also represent an exosome substrate. The exosome could then degrade the RNA and displace Pol II to resolve the R-loop. Again, R-loop unwinding could be facilitated by other factors, for example the helicase SETX (Sen1 in S. cerevisiae).

Table 1: Exosome activating Mtr4-like helicase complexes in different organisms.

\begin{tabular}{|c|c|c|c|}
\hline Organism & nucleoplasm & nucleolus & Reference \\
\hline S. cerevisiae & $\begin{array}{l}\text { Mtr4 within TRAMP } \\
\text { polyA polymerase: Trf4/5 } \\
\text { adds short polyA tails } \\
\text { Zn-knuckle proteins: Air1/Air2 } \\
\text { RNA-binding proteins } \\
\text { substrates: cryptic transcripts, } \\
\text { misprocessed RNA, replication- } \\
\text { dependent histone mRNAs }{ }^{1}\end{array}$ & $\begin{array}{l}\text { Mtr4 within TRAMP } \\
\text { polyA polymerase: Trf4/5 } \\
\text { adds short polyA tails } \\
\text { Zn-knuckle proteins: Air1/Air2 } \\
\text { RNA-binding proteins } \\
\text { substrates: rRNA precursors, } \\
\text { heterochromatic RNA }{ }^{1}\end{array}$ & $14,23,55,119$ \\
\hline S. pombe & $\begin{array}{l}\text { Mtl1 within MTREC } \\
\text { polyA polymerase: - } \\
\text { Zn-knuckle proteins: - } \\
\text { substrates: cryptic transcripts, } \\
\text { misprocessed RNA, transposons, } \\
\text { meiotic mRNAs }\end{array}$ & $\begin{array}{l}\text { Mtr4 within TRAMP } \\
\text { polyA polymerase: Cid14 } \\
\text { adds short polyA tails } \\
\text { Zn-knuckle proteins: Air1 } \\
\text { RNA-binding protein } \\
\text { substrates: rRNA precursors, } \\
\text { heterochromatic RNA }\end{array}$ & $21,25,32,60-62$ \\
\hline human & $\begin{array}{l}\text { hMtr4 within NEXT } \\
\text { polyA polymerase: - } \\
\text { Zn-knuckle proteins: ZCCHC8 } \\
\text { RNA-binding protein } \\
\text { substrates: cryptic transcripts, } \\
\text { misprocessed RNA, transposons }\end{array}$ & $\begin{array}{l}\text { hMtr4 within TRAMP } \\
\text { polyA polymerase: TRF4-2 } \\
\text { adds short polyA tails } \\
\text { Zn-knuckle proteins: ZCCHC7 } \\
\text { RNA-binding protein } \\
\text { substrates: rRNA precursors }\end{array}$ & 18,57 \\
\hline Arabidopsis & $\frac{\text { AtHen }^{2}}{\text { polyA polymerase: - }}$ & $\frac{\text { AtMtr }^{2}}{\text { polyA polymerase: - }}$ & 63 \\
\hline
\end{tabular}


Zn-knuckle proteins: AT5G38600

RNA-binding protein

substrates: cryptic transcripts,

misprocessed RNA, transposons
Zn-knuckle proteins: -

substrates: rRNA precursors

${ }^{1}$ The different targets of nucleoplasmic and nucleolar TRAMP are inferred by localization and homology only.

${ }^{2}$ In Arabidopsis, the complexes that contain Mtr4-like helicases have not been named.

TRAMP - Trf4/5-Air1/2-Mtr4 polyadenylation complex; MTREC - Mtl1-Red1 core; NEXT - nuclear exosome targeting complex

\section{Glossary}

\section{spinal muscular atrophy}

A neuromuscular disorder characterized by degeneration of specific cells within the spinal cord that leads to muscle weakness and atrophy.

\section{pontocerebellar hypoplasias}

A group of severe neurodegenerative disorders that affect growth and function of the brain, resulting in delayed development and frequent early death.

\section{PH-like proteins}

Proteins that resemble the bacterial ribonuclease $\mathrm{PH}$, which is a phosphorolytic exoribonuclease.

\section{DExH-box or DEAD-box}

A short motif that characterizes a family of RNA or DNA-binding, ATP-hydrolysing proteins, many of which are helicases.

\section{non-canonical polyA polymerase}


Member of a conserved subclass of nucleotidyl transferases. Related to the canonical polyA polymerase, which catalyses the addition of polyA tails to mRNAs, but with diverse substrates and functions .

\section{Zn-knuckle}

A zinc-binding structural motif that frequently mediates interactions with nucleic acids.

\section{YTH domain}

A conserved RNA-binding domain named after the splicing factor YT521-B. YTH domains of some proteins specifically recognize methylated adenosines.

\section{Sm ring}

Heptameric protein complex that associates with Sm sites located at the 3' end of snRNAs and telomerase RNA and stabilizes them.

\section{"sno-IncRNAs"}

A class of long ncRNAs that is protected from degradation by the presence of a snoRNA fold. Their biological function is unknown.

\section{SR proteins}

A conserved family of proteins involved in RNA splicing, characterized by a domain with serine and arginine repeats.

\section{cryptic introns}

Introns that are usually spliced at low frequency. Their splicing can be activated in response to external cues and does not necessarily generate a functional transcript. 
(AOA2) A neurodegenerative disorder characterized by juvenile onset of progressive cerebellar ataxia and peripheral neuropathy.

\section{Biographies}

Cornelia Kilchert is a postdoctoral fellow in the laboratory of Lidia Vasiljeva at the University of Oxford, UK, where she studies nuclear RNA metabolism and regulation of gene expression in fission yeast. Her graduate research in the group of Anne Spang at the Biozentrum of the University of Basel, Switzerland, focused on mechanisms of RNA localization and the regulation of RNA metabolism in response to cellular stress.

Sina Wittmann is doing her DPhil in Lidia Vasiljeva's lab at the University of Oxford, UK where she works on understanding the mechanisms of transcriptional interference. Prior to her DPhil she received a Master's degree, for which she carried out research at both the University of Colorado at Boulder, USA and the University of Regensburg, Germany. Lidia Vasiljeva is a Wellcome Trust Senior Research Fellow, located in the Department of Biochemistry at the University of Oxford. She obtained her PhD from the University of Helsinki, Finland studying replication of RNA viruses at the laboratory of Leevi Kääriäinen and did her postdoctoral studies with Steve Buratowski at Harvard Medical School, Boston, USA, where her research was focused on the mechanisms of transcription termination. Her laboratory studies the mechanisms underpinning RNA metabolism in the nucleus. Lidia Vasiljeva’s homepage: http://www.bioch.ox.ac.uk/aspsite/index.asp?pageid=675

\section{Online Summary}

- The nuclear RNA exosome complex is involved in 3' processing of various stable RNA species and is crucial for RNA quality control in the nucleus. It also degrades many types of cryptic transcripts generated as a result of pervasive transcription and removes aberrant RNA molecules that failed to mature properly. Disruption of the RNA exosome or its co-factors is associated with human diseases.

- Targeting substrates to the exosome complex for degradation constitutes a two-step process. Exosome specificity factors recognize and bind certain features on the target RNA and recruit 
activating complexes. Unwinding of the RNA substrate by helicases associated with the activating complexes facilitates RNA degradation by the exosome complex.

- Lack of proper mRNA processing that results in intron retention, transcription read-through, or incorrect mRNP assembly in the absence of packaging factors, induces transcript degradation by the exosome complex.

- RNA surveillance by the exosome co-operates with RNA processing to regulate mRNA levels. Both the induction of non-productive RNA processing (such as premature transcription termination or cryptic splicing) or the suppression of proper mRNA processing (resulting in intron retention or read-through transcription) can be coupled with RNA decay by the exosome complex, thus reducing mRNA levels in response to external cues.

- Several novel functions attributed to the exosome complex include the disassociation of stalled Pol II, and resolving the formation of DNA-RNA hybrids, which are a source of genomic instability. These additional functions appear to be required to enable certain biological processes such as the DNA damage response and antibody class switch recombination.

\section{ToC}

The versatile RNA-degradation functions of the RNA exosome complex render it crucial for RNA biogenesis. It is now emerging that the nuclear exosome is a specific regulator of gene expression in different physiological processes, and has a role in transcription regulation and in maintaining genome stability.

\section{Ontology terms}

Biological sciences/Molecular biology/RNA metabolism/RNA decay [URI /631/337/1645/2020]

Biological sciences/Molecular biology/Transcription [URI /631/337/572]

Biological sciences/Genetics/Genomic instability [URI /631/208/211] 